\title{
SPECT findings in Parkinson's disease associated with dementia
}

Hideyuki Sawada, Fukashi Udaka, Masakuni Kameyama, Naoyuki Seriu, Kazuto Nishinaka, Katsurou Shindou, Mitsuo Kodama, Nobuyuki Nishitani, Kiyohito Okumiya

\begin{abstract}
Dementia in Parkinson's disease is thought to be attributable not only to subcortical lesions but also to cortical alterations, especially frontal lobe dysfunction. To evaluate cortical function, the regional cerebral blood flow (rCBF) was estimated of 13 demented and 13 nondemented age matched patients with Parkinson's disease compared with that of 10 age matched controls using I-123 iodoamphetamine single photon emission tomography (IMP-SPECT). The rCBF of the nondemented Parkinson's patients showed no significant differences from that of the control subjects. In the demented patients, the bilateral frontal and parietal and left temporal regional blood flow was significantly less than in the controls. Four demented patients showed isolated frontal hypoperfusion, 8 showed fronto-parietal hypoperfusion, and 1 showed isolated parietal hypoperfusion. Frontal hypoperfusion was therefore present in 12 of the 13 demented patients, and this finding agrees with the frontal lobe dysfunction hypothesis. Parietal rCBF had a significant positive correlation with cortical functions such as calculation and language ability in the MMSE scores. The parietal and temporal reduction in $\mathrm{TCBF}$ probably reflects the presence of Alzheimer pathology, cortical Lewy body disease, or both.
\end{abstract}

(F Neurol Neurosurg Psychiatry 1992;55:960-963)

The prevalence of dementia in Parkinson's disease ranges from $14 \%-40 \%$ in reported series. ${ }^{1}$ This wide variation is related to differences in the diagnostic criteria used or the methods adopted by various investigators. Follow up studies using DSM-III-R diagnostic criteria for dementia have shown a prevalence of $10.9-23.6 \%$ in Parkinson's disease patients. ${ }^{23}$ The intellectual dysfunction is generally of the subcortical type, with the slowing of mental processing and the development of forgetfulness, depression and apathy. Pathological studies have shown that the subcortical nuclei, such as the substantia nigra and locus coeruleus, are affected in Parkinson's disease patients with dementia. ${ }^{4}$ As in other types of subcortical dementia, such as progressive supranuclear palsy, the frontal lobes are also thought to be affected in patients with dementia due to Parkinson's disease. Secondary frontal lobe changes may occur because of the connections between this lobe and the various subcortical nuclei (frontal lobe dysfunction hypothesis). ${ }^{5}$

In addition, $17 \%$ of Parkinson's disease patients show Alzheimer changes, similar to the prevalence of dementia in Parkinson's disease. All Parkinson's disease patients have a few cortical Lewy bodies at necropsy, but only a count above 5 per high powered field is consistently associated with dementia. Both coincidental Alzheimer pathology and cortical Lewy bodies have been shown to be associated with dementia in Parkinson's disease.

It therefore remains uncertain whether dementia in Parkinson's disease can be attributed to subcortico-frontal lobe dysfunction alone, or whether it also involves widespread cortical dysfunction.

I-123 iodoamphetamine single photon emission tomography (IMP-SPECT) is a practical modality for the study of changes in rCBF which reflect cortical function. To discover cortical function in dementia of Parkinson's disease, we studied the clinical features of 26 Parkinson's disease patients and correlated them with the regional cerebral blood flow as determined by IMP-SPECT.

\section{Subjects and methods}

The subjects included 13 demented and 13 nondemented age matched patients with Parkinson's disease who were randomly selected from those admitted to Sumitomo Hospital from 1989-91.

The DSM-III-R diagnostic criteria for dementia were used to assess the subjects. Evidence of memory impairment plus aphasia, impaired judgment, impaired visuospatial skills, or inability to perform complex ideational tasks was necessary to meet the criteria for dementia. We excluded patients with active delirium or confusion. The diagnosis of Parkinson's disease was based upon the following clinical features, cogwheel-type muscular rigidity, tremor, bradykinesia, an initial response to levodopa, and a chronic progressive course over longer than one year. Patients who had been administered neuroeptics were excluded. We also excluded patients given more than $4 \mathrm{mg}$ of trihexyphenidyl from the demented group. We used MRI to rule out vascular dementia and vascular Parkinsonism. We also excluded patients with a poor drug response or putaminal atrophy on MRI because of a possible diagnosis of striato-nigral 
degeneration. Furthermore, patients suspected of having progressive supranuclear palsy, who showed a poor response to drug therapy and had severe disturbances of postural reflex or supranuclear ophthalmoplegia, were also excluded. Motor disability (including rigidity, tremor, and bradykinesia) was evaluated as absent (0), mild (1), moderate (2), or severe (3) by neurological examination. Overall disability was assessed according to the scale of Hoehn and Yahr.

In the demented patients, we investigated orientation, registration, calculation, recall, language ability, and praxis using the MiniMental State Examination (MMSE). ${ }^{6}$ Behavioural disturbances, such as impulsive behaviour, mental irritability, and a history of delirium resistant to medical treatment, were assessed by interview and by review of the medical records.

The rCBF was measured by IMP-SPECT using images with a resolution of $15 \mathrm{~mm}$. The tracer was injected as a $5-\mathrm{mCi}$ intravenous bolus; scanning began 20 minutes after injection and continued for 60 minutes. Patients kept their eyes closed during scanning and rested in a quiet room. Transaxial SPECT images were taken parallel to the orbitomeatal line at the following levels; upper ventricular, midventricular, midtemporal, and midcerebellar hemispheric. The region of interest (ROI) used was a $21 \mathrm{~mm} \times 21 \mathrm{~mm}$ square. We established ROIs in the bilateral frontal, parietal (upper ventricular level), occipital (midventricular level), and temporal (midtemporal level) lobes, and in the cerebellar hemispheres (midcerebellar level) (fig 1). The relative rCBF of each ROI was determined by comparison with the mean cerebellar rCBF as follows:

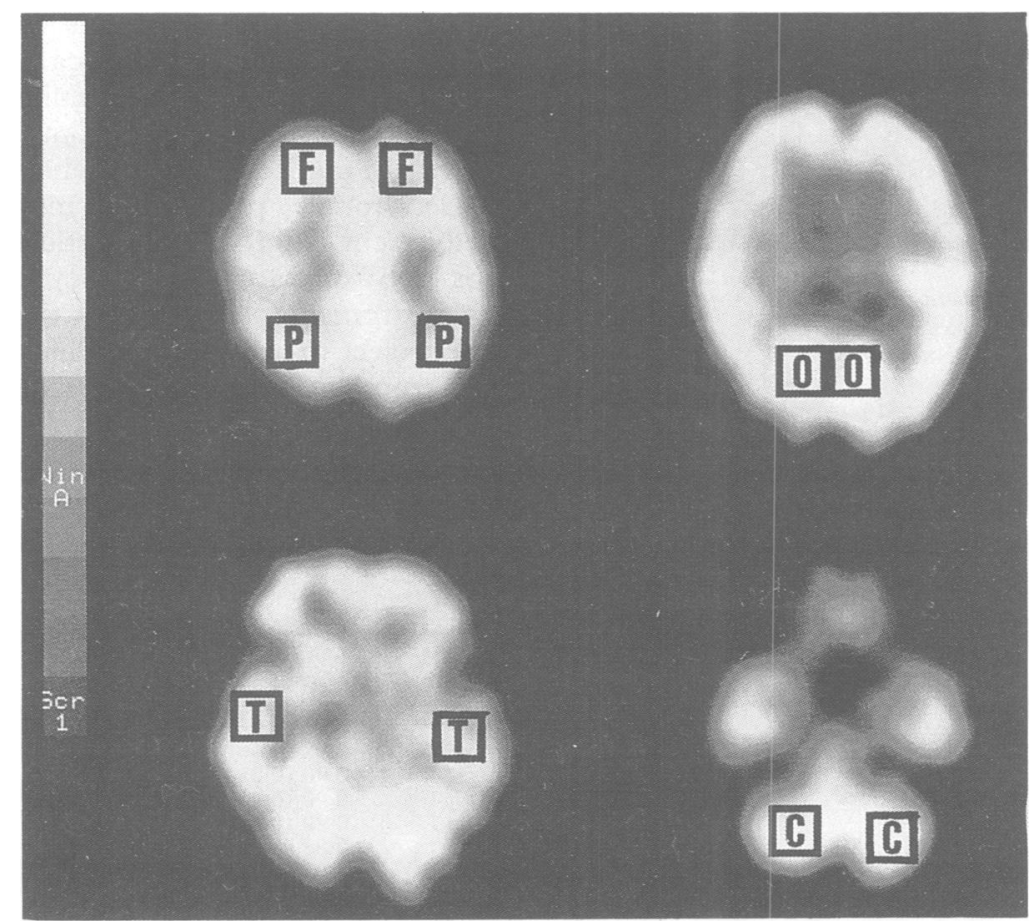

Figure 1 Horizontal sections of IMP-SPECT images at the upper ventricular, midventricular, midtemporal, and midcerebellar levels. Regions of interest were set in the frontal $(F)$, parietal $(P)$, temporal $(T)$, and occipital $(O)$ lobes, and in the cerebellar hemispheres $(C)$.
Relative $\mathrm{rCBF}=2 \times(\mathrm{rCBF}) \mathrm{ROI} /$
$((\mathrm{rCBF}) \mathrm{rc}+(\mathrm{rCBF}) \mathrm{lc})$

(rc, lc: right and left cerebellar hemispheres)

Patients were only enrolled after informed consent was obtained and after a detailed description of the risks and benefits of the study was provided.

We compared mean duration of disease and the severity of muscular rigidity, tremor, and bradykinesia between the demented and nondemented patients. Mean duration of disease was compared using the normal Student's $t$ test, while the severity of muscular rigidity, tremor, and bradykinesia were compared using one-way ANOVA.

The rCBF of the demented and nondemented patients was compared with that of healthy controls. These control subjects were 10 age matched normal volunteers. Mean values were analysed using the normal Student's $t$ test when there was no significant difference in the variances, or using Welch's $t$ test when there was a significant difference in variance. In the demented patients, we analysed the correlation between $\mathrm{rCBF}$ and the MMSE score using Spearman's rank correlation coefficients.

\section{Results}

Comparison of the duration of disease, and the severity of muscular rigidity, tremor, and bradykinesia showed no significant differences between the demented and nondemented groups (table 1). The MMSE scores for the demented patients ranged from 2 to 25 (mean $=17 \cdot 4$, standard deviation $=6 \cdot 80$ ). In the patients with better preserved MMSE scores, severe behavioural disturbances such as mental irritability or abnormal impulsive behaviour affected their daily lives.

The rCBF distribution of the Parkinson's patients without dementia showed no significant difference from that of the controls. In the demented patients, the rCBF of the bilateral frontal, parietal, and left temporal lobes was significantly reduced compared with the controls. However, the rCBF in the other ROIs did not show any significant differences (table 2).

With a 2SD criterion, 7 out of the 13 demented patients had significantly reduced

Table 1 A comparison of clinical features between demented and nondemented patients with Parkinson's disease

\begin{tabular}{|c|c|c|c|}
\hline & $\begin{array}{l}\text { Demented } \\
(n=13) \\
\text { Mean }(S D)\end{array}$ & $\begin{array}{l}\text { Nondemented } \\
(n=13) \\
\text { Mean }(S D)\end{array}$ & $\begin{array}{l}\text { Controls } \\
(n=10) \\
\text { Mean }(S D)\end{array}$ \\
\hline $\begin{array}{l}\text { Age (y) } \\
\text { Sex (M/F) } \\
\text { Duration (y) } \\
\text { Yahr (1-5) } \\
\text { Rigidity (0-3) } \\
\text { Bradykinesia (0-3) } \\
\text { Tremor (0-3) }\end{array}$ & $\begin{array}{l}72 \cdot 0(9 \cdot 2) \\
8 / 5 \\
6 \cdot 1(4 \cdot 4) \\
3 \cdot 2(0 \cdot 8) \\
1 \cdot 4(1 \cdot 0) \\
1 \cdot 7(0 \cdot 9) \\
0 \cdot 9(1 \cdot 0)\end{array}$ & $\begin{array}{l}68 \cdot 6(6 \cdot 4) \\
6 / 7 \\
7 \cdot 6(4 \cdot 8) \\
3 \cdot 2(0 \cdot 7) \\
1 \cdot 5(0 \cdot 5) \\
1 \cdot 6(0 \cdot 9) \\
1 \cdot 0(0 \cdot 8)\end{array}$ & $\begin{array}{l}71 \cdot 4(7 \cdot 5) \\
5 / 5 \\
\text { NS a } \\
\text { NS b } \\
\text { NS c } \\
\text { NS d } \\
\text { NS e }\end{array}$ \\
\hline
\end{tabular}

NS; not significant

a: Student's $t$ test ( $\mathrm{df}=24)$, to $=0.85$

b-e: ANOVA (df1 $=1, \mathrm{df2}=24$ )

Fo $(b)=0.07$, Fo $(c)=0.26$, Fo $(d)=0.05$, Fo $(e)=0.04$ 


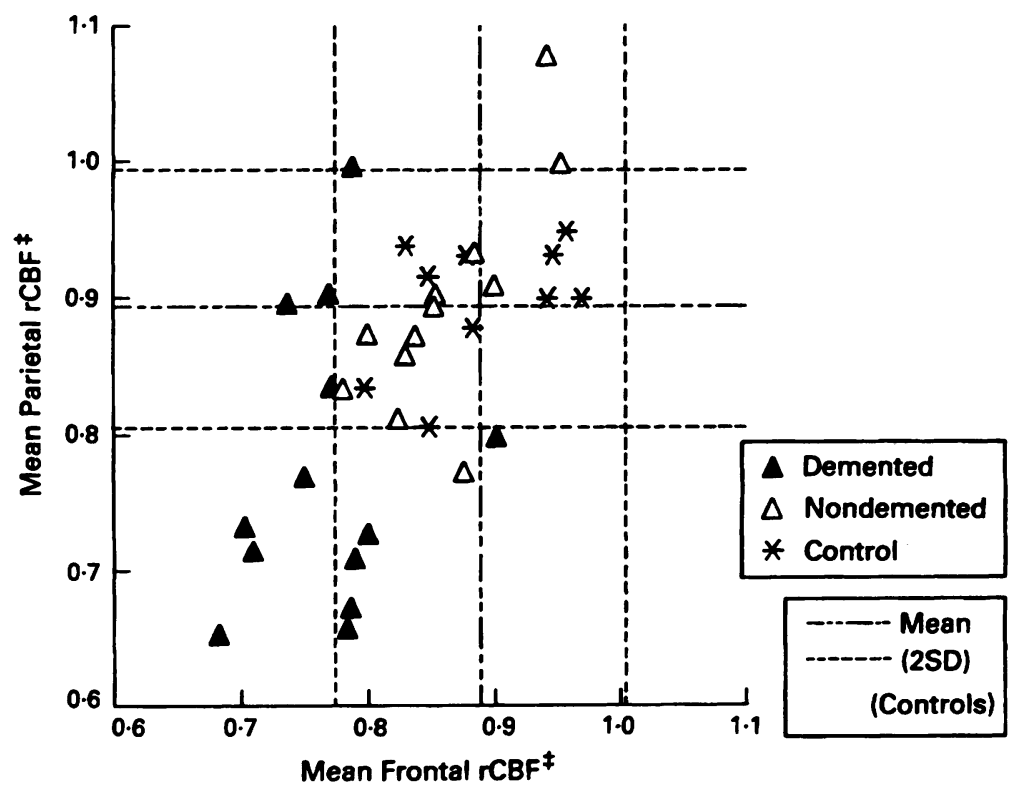

Figure 2 Scattergram of mean frontal and parietal rCBF. Asterisks indicate normal controls. Closed triangles indicate the 13 demented patients. Open triangles indicate the 13 nondemented patients. Almost all nondemented patients had a distribution similar to that of the controls. All demented patients (except one) had a frontal blood flow of less than 0.8. The mean parietal blood flow was more than 0.81 (2SD below the normal mean parietal $r C B F$ ) in 4 patients (frontal hypoperfusion in fig 3), and less than 0.81 in 8 patients (fronto-parietal hypoperfusion pattern in fig 3).



Figure 3 IMP-SPECT patterns in 1 nondemented (left) and 2 demented (centre and right) patients with Parkinsons's disease. The upper image is a transaxial view at the upper ventricular level and the lower image is a parasagittal view. The nondemented patient showed only slight frontal hypoperfusion (left), while the demented patients showed marked hypoperfusion of the frontal lobes (centre), or of both the frontal and parietal lobes (right). Four of the 13 demented patients studied had the IMP-SPECT pattern shown in the centre of the figure, and 8 had the pattern shown on the right. frontal rCBF, 9 had reduced parietal rCBF, and 12 had one or both (fig 2). The cerebral blood flow distribution showed at least 2 patterns, an isolated reduction in frontal flow (4 patients) and a reduction in both frontal and parietal flow (8 patients). Reduction of blood flow in the frontal lobes was predominantly seen in the association area rather than in the motor and pre-motor areas (fig 3).

Analysis of the relationship between $\mathrm{rCBF}$ and the MMSE score showed that the left parietal rCBF had a significant positive correlation with the scores for registration ( $\mathrm{rs}=$ $0.57, \mathrm{p}<0.01(\mathrm{df}=24$, to $=3.44)$ ), calculation ( $\mathrm{rs}=0.41, \mathrm{p}<0.05$ (to $=2.18)$ ), and language ( $\mathrm{rs}=0.60, \mathrm{p}<0.01$ (to $=$ 3.66)).

The patients with isolated frontal hypoperfusion did not develop delirium. In contrast, 4 of the 9 patients with fronto-parietal hypoperfusion had a history of severe delirium, and these 4 patients showed more severe cortical intellectual deterioration (mean MMSE score: 11, range: 2-16).

\section{Discussion}

Previous reports on cerebral blood flow in Parkinson's disease ${ }^{7-10}$ showed no abnormalities or only slight hypometabolism in the frontal area in patients without dementia, and we found a similar pattern in our nondemented patients. In contrast, the frontal hypoperfusion seen in the demented group suggested that alterations had occurred in the cortex or in the subcortical nuclei projecting to the frontal lobes.

In Parkinson's disease, subcortical degeneration leading to dementia involves not only the dopaminergic system arising from the substantia nigra but also the basal cholinergic system arising from the nucleus basalis of Meynert and the diagonal band of Broca. ${ }^{11}$ Damage to these subcortical nuclei projecting to the frontal cortex could lead to the frontal hypoperfusion seen in our demented patients.

The subcortico-frontal projections may include two different nigro-striato-cortical loops, a "motor loop" and a "complex loop". Neurons in the rostromedial compacta of the substantia nigra project to the caudate, which connects to the frontal association area (the "complex loop"), while neurons in the caudolateral compacta project to the putamen and

Table 2 A comparison of $r C B F$ between demented and nondemented patients

\begin{tabular}{llll}
\hline & $\begin{array}{l}\text { Demented }(n=13) \\
\text { Mean }(S D)\end{array}$ & $\begin{array}{l}\text { Nondemented }(n=13) \\
\text { Mean }(S D)\end{array}$ & $\begin{array}{l}\text { Controls }(n=10) \\
\text { Mean }(S D)\end{array}$ \\
\hline Rt Frontal & $0.78(0.06) \mathrm{a}$ & $0.86(0.05)$ & $0.90(0.06)$ \\
Lt Frontal & $0.76(0.06) \mathrm{b}$ & $0.85(0.06)$ & $0.88(0.06)$ \\
Rt Parietal & $0.78(0.12) \mathrm{c}$ & $0.89(0.09)$ & $0.90(0.05)$ \\
Lt Parietal & $0.77(0.10) \mathrm{d}$ & $0.89(0.08)$ & $0.90(0.04)$ \\
Rt Temporal & $0.87(0.07)$ & $0.89(0.10)$ & $0.89(0.05)$ \\
Lt Temporal & $0.83(0.05)$ & $0.86(0.07)$ & $0.88(0.05)$ \\
Rt Occipital & $1.02(0.10)$ & $1.05(0.05)$ & $1.07(0.05)$ \\
Lt Occipital & $1.06(0.10)$ & $1.08(0.07)$ & $1.07(0.04)$ \\
\hline
\end{tabular}

a-d; Significantly reduced compared with controls (un-paired, two-tailed t-test)

a (df $=21$, to $=4.67) \mathrm{p}<0.001$ (normal t-test)

b (df $=21$, to $=4.63) \mathrm{p}<0.001$ (normal t-test)

$c(\mathrm{df}=17$, to $=3.25) \mathrm{p}<0.01$ (Welch method t-test)

$\mathrm{d}(\mathrm{df}=17$, to $=4.05) \mathrm{p}<0.001$ (Welch method $\mathrm{t}$-test). 
then connect to the pre-motor and motor areas (the "motor loop"). The "complex loop" is related to psychomotor function and motivation, while the "motor loop" is related to motor function. ${ }^{12}$ The predominance of blood flow changes in the frontal association cortex may thus reflect alterations in the "complex loop" due to neuronal loss in the medial substantia nigra. ${ }^{13}$

In addition to the nigro-striato-cortical loops, dopaminergic cells in the ventral tegmental area of the midbrain project to the frontal lobe, ${ }^{14}$ and destruction of this area causes behavioural disturbance. ${ }^{15}$ In Parkinson's disease, these cells are also involved. ${ }^{16}$ Thus the frontal hypoperfusion, that we found in 12 out of 13 demented patients could reflect disturbances of various subcortical nuclei projecting to the frontal cortex (the frontal lobe dysfunction hypothesis).

In addition to frontal hypoperfusion, parietal and left temporal rCBF showed significant reductions compared with the controls. The rCBF in these regions had a significant positive correlation with cortical function such as language and calculating ability. The cortical alterations associated with dementia in Parkinson's disease include certain pathological findings also seen in Alzheimer's disease. A combination of the patholonical changes seen in both Parkinson's disease and Alzheimer's disease occurs in $16.9 \%$ of such patients ${ }^{17}$ and the topographical distribution of Alzheimer's pathology in Parkinson's disease patients is similar to that in pure Alzheimer's disease. In Alzheimer's disease, rCBF studies with SPECT have revealed hypoperfusion in the parietal lobes. ${ }^{18}$

Another cortical change associated with dementia in Parkinson's disease is the occurrence of Lewy bodies. The combination of Lewy bodies and senile plaque in the cortex is seen in diffuse Lewy body disease, which is frequently accompanied by acute delirium or agitation and cortical type dementia. ${ }^{19-21}$ In this study, 4 patients with parietal hypoperfusion also showed nocturnal delirium and cortical dementia, and these clinical features coincided with those of diffuse Lewy body disease. Cortical Lewy bodies are generally numerous in the frontal, temporal, and parietal lobes, ${ }^{22}$ and the SPECT patterns of these patients could reflect the distribution of Lewy bodies. It still remains to be determined whether the cortical lesions of such patients with parietal hypoperfusion are due to $\mathrm{Alz}$ heimer's pathology or Lewy bodies.

We conclude that SPECT images of demented Parkinson's disease patients show at least 2 patterns-isolated frontal lobe hypo- perfusion or hypoperfusion in both the frontal and parietal areas. In our series, frontal lobe hypoperfusion was the common finding in demented Parkinson's disease, data which is coincident with the frontal lobe dysfunction hypothesis. In the patients with fronto-parietal hypoperfusion, not only frontal lobe deficits but also more widespread cortical dysfunction may contribute to their dementia.

1 Mayeux R. Depression and dementia in Parkinson's disease. In: Marsden CD, Fahn S, eds. Movement disorders. Woburn, Mass: Butterworths, 1982:75-95.

2 Mayeux R, Stern Y, Rosenstein R, et al. An estimate of the prevalence of dementia in idiopathic Parkinson's disease. prevalence of dementia in idiop
Arch Neurol 1988;45:260-2.

3 Ebmeier KP, Calder SA, Crawford JR, et al. Clinical features predicting dementia in idiopathic Parkinson' disease: a follow up study. Neurology 1990;40:1222-4.

4 Gaspar P, Gray F. Dementia in idiopathic Parkinson's disease. A neuropathological study of 32 cases. Acta Neuropathol 1984;64:43-52.

5 Taylor AE, Saint-Cyr JA, Lang AE. Frontal lobe dysfunction in Parkinson's disease. Brain 1986;109:845-83.

6 Folstein MF, Folstein SE, McHugh PA. Mini-Mental State: a practical method for grading the cognitive state of patients for the clinician. $f$ Psychiatr Res 1975;12: 189-98.

7 Kul DE, Metter EJ, Riege WH. Patterns of local cerebral glucose utilization determined in Parkinson's disease by the 18F fluorodeoxyglucose method. Ann Neurol 1984; 15:419-24.

8 Henriksen L, Boas J. Regional cerebral blood flow in hemiparkinsonian patients. Emission computerized tomography of inhaled 133 xenon before and after evodopa. Acta Neurol Scand 1985;71:257-66

9 Perlmutter JS, Raichle ME. Regional blood flow in hemiparkinsonism. Neurology 1985;35:1127-34.

10 Wolfson LI, Leenders KL, Brown LL, Jones T. Alterations of regional cerebral blood flow and oxygen metabolism in Parkinson's disease. Neurology 1985;35:1399-405.

11 Whitehouse PJ, Hedreen JC, White CL III, Price DL. Basal foebrain neurons in the dementia of Parkinson's disease. Ann Neurol 1983;13:243-8.

12 Hornykiewicz O, Kish SJ. Biochemical pathophysiology of Parkinson's disease. In: Yahr MD, Bergmann KJB, eds. Advances in neurology, vol 45. New York: Raven Press, Advances in

13 Rinne JO, Rummukainen J, Paljarvi L, Rinne UK. Dementia in Parkinson's disease is related to neuronal loss in the tia in Parkinson's disease is related to neuronal loss in

14 Lindvall O, Bjorklund A. Organization of catecholamine neurons in the rat CNS. In: Iversen LI, et al, eds. Handbook of psychopharmacology, vol 9. New York: Plenum, 1978:139-231

15 Le Moal M, Galey D, Cardo B. Behavioural effects of local injection of 6-hydroxydopamine in the medial ventral egmentum in the rat. Possible role of mesolimbic dopaminergic systerm. Brain Res 1975;88:190-4.

16 Javoy-Agid F, Taquet H, Ploska A, Cherif-Zahar C, Ruberg $M$, Agid Y. Distribution of catecolamines in the ventral mesencephalon of human brain, with special reference to Parkinson's disease. $\mathcal{f}$ Neurochem 1981;36:2101-5.

17 Jellinger K. Overview of morphological changes in Parkinson's disease. In: Yahr MD, Bergmann KJB, eds. Advances in Neurology, vol 45. New York: Raven Press, in Neurolo

18 Johnson KA, Mueller ST, Walshe TM, English RJ, Holman L. Cerebral perfusion imaging in Alzheimer's disease. Use of single photon emission computed tomography and iofetamie hydrochloride I 123. Arch Neurol 1987;44: 165-8.

19 Crystal HA, Dickson DW, Lizardi JE, Davies P, Wolfson LI Antemortem diagnosis of diffuse Lewy body disease. Neurology 1990;40:1523-8.

20 Byrne E, Lennox G, Lowe J, Godwin-Austen RB. Diffuse Lewy body disease: clinical features in 15 cases. $\mathcal{f}$ Neurol Neurosurg Psychiatry 1989;52:709-17.

21 Perry RH, Irving D, Blessed G, Perry EK, Fairbairn AF Clinically and neuropathologically distinct form of dementia in the elderly. Lancet 1989;1:116.

22 Gibb WRG, Esiri M, Lees AJ. Clinical and pathological features of diffuse cortical Lewy body disease (Lewy body dementia). Brain 1985;110:1131-53. 